\title{
Community-Based Progressive Aquatic Exercise for the Management of Knee Osteoarthritis
}

\author{
Dayle Maryanna Masslon, ${ }^{1,}$ Aki Takahashi, ${ }^{1}$ Allison Rodgers, ${ }^{1}$ Amelia Foster, ${ }^{1}$ Megan Kennedy, ${ }^{1}$ Steve \\ Victorson, ${ }^{1}$ Michael Joseph, ${ }^{1}$ and Craig R Denegar ${ }^{1}$ \\ ${ }^{1}$ Physical Therapy, University of Connecticut, Storrs, United States \\ "Corresponding author: Dayle Maryanna Masslon, Physical Therapy, University of Connecticut, Storrs, United States. Tel: +1-8608828109, Fax: +1-8604566956, E-mail: \\ masslond@gmail.com
}

Received 2016 July 12; Revised 2016 September 16; Accepted 2016 September 22.

\begin{abstract}
Background: We examined the feasibility and effectiveness of a community-based progressive aquatic exercise program for community dwelling older adults, with moderate to severe knee osteoarthritis (OA).

Objectives: The purposes of this study were to 1, assess the effects of a progressive aquatic exercise program on the walking ability, stair climbing ability, quadriceps muscle strength, as well as self-reported symptoms, function, and quality of life in community dwelling adults with moderate to severe knee $\mathrm{OA}$ and; 2 , assess the feasibility of a community-based aquatic program for community dwelling adults with knee OA.

Methods: Seventeen volunteers ( 12 women ( $\mathrm{x}=61.1$ years) and 5 men $(\mathrm{x}=69.0$ years $)$ ) participated in a progressive $8-10$ week aquatic exercise program, consisting of 20 - 24, 1-hour sessions. Outcome measures, acquired twice before beginning the exercise protocol as well as after 4 and 8 weeks of exercise, included the Knee Injury and Osteoarthritis outcome score (KOOS) instrument, a 2 minute walk test (2MWT), a 10 step stair climb for time, and an isometric knee extension strength assessment.

Results: Significant improvements were detected in 2 MWT, 10 step stair climb, right quadriceps isometric force development, and the KOOS symptoms and stiffness subscale. Significant improvement was found on KOOS function subscales between baseline testing sessions and maintained at follow-up. Non-significant improvements were identified in left quadriceps isometric force development, KOOS pain, and KOOS quality of life.

Conclusions: These data suggest that a community-based, progressive aquatic exercise program is feasible and results in measurable improvements in function without worsening symptoms. Further study is warranted to investigate the impact of a longer program and the role of aquatic exercise in the long-term management of patients with knee OA.
\end{abstract}

Keywords: Knee, Osteoarthritis, Progressive, Aquatic, Exercise

\section{Background}

Knee osteoarthritis (OA) affects more than 7.7 million people in the United States (1) and $37.4 \%$ of adults over the age of 60 years. OA of the knee, hip, and hand is more common in women (2), with knee OA affecting $42.1 \%$ of females and $31.2 \%$ of males over the age of 60 (3). OA is a progressive disease, which occurs with the degeneration of articular cartilage, joint lining, ligaments, and bone. Hyaline cartilage is avascular, aneural, and lacks perichondrium resulting in an extremely low healing capacity. OA can be radiographically staged using the Kelgren-Lawrence scale, which assesses the extent of degeneration, including decreased joint space, osteophyte development, and sclerosis.

The Osteoarthritis research society international
(OARSI) guidelines for the non-surgical management of knee OA, identified core components of intervention to include weight management, education, and strengthening (4). Strengthening can be achieved through land or aquatic-based exercise programs. Strong evidence supports therapeutic exercise as the core component of conservative management of knee OA (5). Aquatic exercise is recommended by OARSI as well as and by the American college of rheumatology (ACR) $(4,6)$ as a non-pharmacological method of controlling knee osteoarthritis symptoms. Both land-based and aquatic exercise programs have advantages and disadvantages that must be considered for each patient. Land-based exercise has been shown to effectively increase strength, reduce knee pain, and improve physical function in the short term setting (5). Land-based programs are easily 
accessible, as many exercises can be performed almost anywhere. Land-based exercises, however, can result in muscle soreness and increased joint pain and swelling. Aquatic exercise introduces a mode of strengthening with less joint loading and potentially less joint and muscle irritation. Access to aquatic exercise programs is more limited and many aquatic exercise programs are not designed to progress at an appropriate intensity required for strengthening. Research addressing the effectiveness of a progressive aquatic protocol is limited.

\section{Objectives}

The purposes of this study were to 1 , assess the effects of a progressive aquatic exercise program on the walking ability, stair climbing ability, quadriceps muscle strength, as well as self-reported symptoms, function, and quality of life in community dwelling adults with moderate to severe knee OA and; 2 , assess the feasibility of a communitybased aquatic program for community dwelling adults with knee OA.

\section{Methods}

\subsection{Participants}

Following approval by the University of Connecticut institutional review board for the protection of human subjects, participants were recruited through flyers distributed at Swymfit Inc., as well as neighboring physician and physical therapy practices. Women and men over the age of 18 seeking to participate were provided information about the study and asked to contact their personal physician. Potential participants were informed that they were not eligible to participate if they had received a hyaluronic acid or corticosteroid injection in either knee in the previous 6 months. Those who received medical clearance by their physician to participate and had physician reported grade II or III knee OA (Kelgren-Lawrence scale) were enrolled. In participants with bilateral knee OA, the selfselected worse knee was used for self-report assessment. See Figure 1 for schematic of the research design. 20 subjects were initially enrolled.

Participants who met the inclusion criteria and received medical clearance then provided informed consent and received a detailed description of the exercise protocol. Participants completed testing at intake (Baseline 1), 1 - 3 weeks later at the first exercise session (Baseline 2), midway (M), and at the final exercise session (F). Participants were asked to maintain a log of medications and general physical activity on a weekly basis. Subjects were permitted to continue any current medications.

\subsection{Outcome Measures}

Testing consisted of the following: The knee injury and osteoarthritis outcome score (KOOS score) - A likert version of the KOOS instrument was used with scored responses to each element of the KOOS ranging from 0 - 4. The instrument has 5 domains: pain (9 questions), symptoms and stiffness (7 questions), function-activities of daily living (16 questions), function- sport and recreation (5 questions), and knee related quality of life (QOL) (4 questions). Scores for global and domain categories were transformed into a total score ranging 0 - 100. A score of 0 indicates extreme knee problems, whereas a score of 100 indicates no knee problems. The internal consistency for the KOOS is 0.86 0.96 and test-retest reliability (ICC) is generally good to excellent (0.67 - 0.95) (7).

2 minute walk test - The 2 minute walk test is an efficient measure of functional community ambulation capacity (8). We measured the maximum distance walked in 2 minutes on a 75 foot concrete indoor course with markers at every 5 feet. Participants were instructed walk as far as possible at as quick a pace as they were comfortable with. Standing rest was allowed if necessary, although none of the participants required a rest period.

10 steps stair climb-lower limb function was measured using a 10 step stair ascending test. The participants were instructed to climb 10 steps as quickly as they could twice at each measurement session. None of the participants required an assistive device, but each was instructed that they could use the side railings if needed. Two recorders recorded the time needed to climb 10 steps and plant both feet on the top step. The mean of the four values was used for data analysis. The reliability of measures between testers in each baseline 1 trials was excellent $\operatorname{ICC}(3,1)=0.95$ and standard error of measurement acceptable (SEM $=0.31$ and 0.29 seconds) The stair height at the Swymfit facility was $14.2 \mathrm{~cm}$.

Isometric knee extension strength- quadriceps strength was measured at approximately 70 degrees of knee flexion. Participants sat on a knee extension machine with the knee flexed and resistance arm locked in place. The resistance arm was used to stabilize a handheld dynamometer (Handheld Dynnamometer (HHD)- MicroFET2 HHD from Hoggan health industries, West Jordan, UT, USA ). Testers were trained in the use of the handheld dynamometer prior to testing based on the methods described by Bohannon et al. (9). Bohannon et al. reported good to excellent reliability with a similar set-up of belt stabilized hand-held dynamometry (9).

\subsection{Intervention}

Participants completed a 1 hour progressive aquatic exercise program (described in Table 1), 2 - 3 times per week 


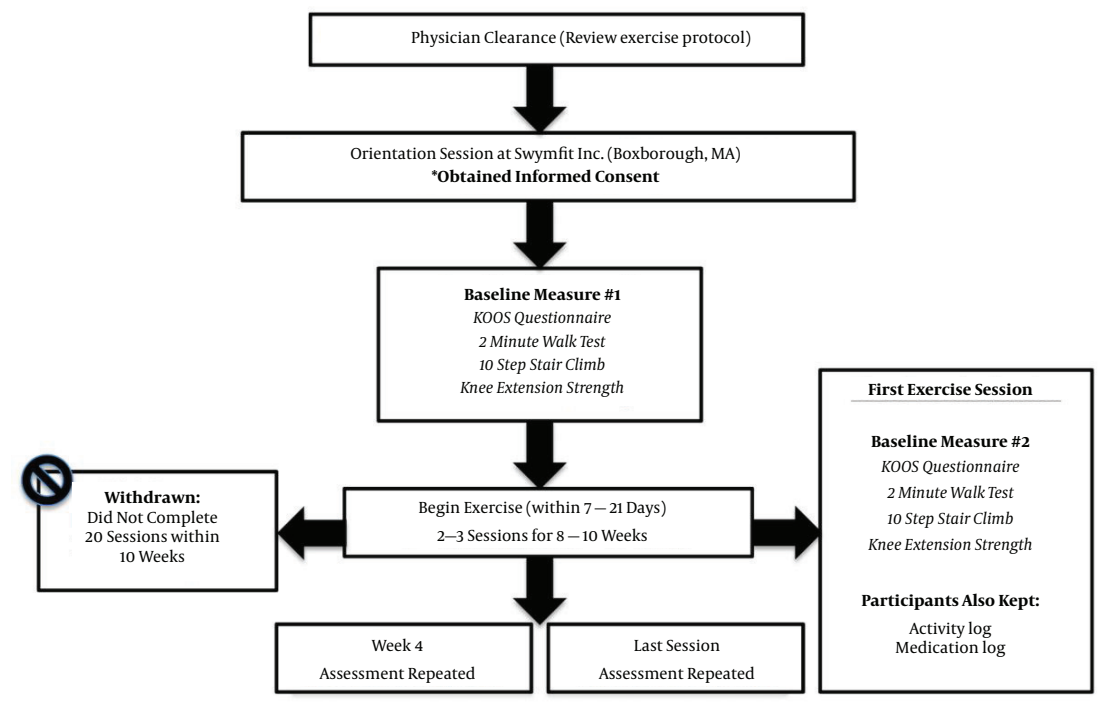

Figure 1. Study Design

for 8 - 10 weeks. The combination of exercises and progression were adapted from programs developed by one of the authors (SV) for programming at the Swymfit Inc. (Boxborough MA, USA) facility for sports rehabilitation and training. The exercises follow normal movement patterns of the lower extremity and the seated leg kicks, forward and backward imitate basic leg extension and hamstring curl machines. Progression was based on individual tolerance and clinical judgment that is consistent with supervised rehabilitation.

Participants were required to complete at least $20 \mathrm{ex}$ ercise sessions within 10 weeks for inclusion in data analysis. The exercise protocol included use of the following equipment: A, Hydrofit classic wave belt to correct body alignment by balancing buoyancy around the midsection; B, Hydrofit mini-cuffs introduced in week 5 , to provide added resistance in the water and increase hip and knee ROM secondary to added buoyancy at the ankle with the leg extended; C, Hydrofit sports therapy barbells (Hydrofit pool equipment- HYDRO-FIT, Eugene, OR, USA) for support and added resistance for stabilization exercises; D, Burdenko Water walkers (Burdenko Water Walkers- Burdenko water and sports therapy institute, Newton, MA, USA) introduced by week 7, to mimic walking up and down stairs to improve community ambulation and function along with resistance training with both lower extremity flexion and extension; E, rectangular swim boards used for seated exercises. Hydrofit collars (Hydrofit pool equipment- HYDRO-FIT, Eugene, OR, USA) were made available to participants to alleviate stress on the neck area if necessary. Exercise progression was individualized, based on self-reported ability to progress, and ultimately reaching $70 \%-80 \%$ of self-perceived maximal effort. Participants were asked about fatigue and soreness experienced in the previous exercise session. Progression was encouraged when there were minimal complaints, which was typical throughout the exercise program. All sessions were conducted in the deep end of the swimming pool $(2.74 \mathrm{~m})$ at the Swymfit Inc. facility.

\subsection{Statistical Methods}

Data were analyzed using SPSS version 20 software. In two instances of missing data due to incomplete KOOS forms not detected at follow-up, scores were replaced using the last value carried forward. This represents a conservative estimate that assumes no additional improvement over the final 4 weeks of the study. Repeated measures ANOVA was completed for the 2-minute walk, stair climb, quadriceps strength and KOOS subsection data. In circumstances where the assumption of sphericity was not met (stair climb, right leg extension force, ADL function, and QOL) Greenhouse-Geisser correction was employed to provide an exact P-value. Planned comparisons were conducted and effect size calculated. We elected not to control for multiple comparisons due to the exploratory nature of the study and the unique construct of each measure. The 2-minute walk test is a measure of endurance, the 10 step stair climb tests function and power, isometric knee extension strength measures quadriceps muscle strength, and 
the KOOS is a self-report of pain, symptoms and stiffness, ADL function, sport and recreational function, and QOL.

\section{Results}

Twenty participants were initially enrolled in the study. Three participants withdrew from the study due to scheduling and medical concerns not related to the study. Twelve women (age $61.1 \pm 6.3$ years, 7 grade III and 5 grade II $\mathrm{OA}$ ) and 5 men (age $69.0 \pm 3.3$ years, 3 grade III and 2 grade II OA) completed 20 - 24 exercise sessions. Baseline performance measures are found in Table 2. None of the participants reported a change in or increased consumption of any medications related to their osteoarthritis during the study. Three reported a daily regimen of non-steroidal antiinflammatory medication once $(\mathrm{n}=1)$, or twice daily $(\mathrm{n}=$ 2 ) with the remainder reporting occasional use of medication for knee pain on an as-needed basis.

\subsection{Outcome Measures}

Participants experienced a significant increase in 2minute walk (large effect (1. 1), mean $=23.4 \mathrm{~m}$. 95\% CI $=$ 12.6-37.3, $\mathrm{P} \leq 0.01)$ performance. Participants experienced a significant decrease in time required to climb 10 steps (large effect (0.86), mean $=1.21$ seconds, 95\% CI $0.53-1.88, \mathrm{P}$ $<0.01)$. Knee extensor strength decreased between the 1st and 2nd baseline test, returned to baseline by 4 weeks and improved significantly from baseline (small effect (0.18), mean $=23.9 \mathrm{~N}, 95 \% \mathrm{CI} 10.2-37.6, \mathrm{P}=0.05)$ on the right and non-significantly on the left ( $\operatorname{small}$ effect $(0.16)$, mean $=16.7$ $\mathrm{N}, 95 \%$ CI $51.3--18.1, \mathrm{P}=0.32$ ). See Figure 2. Increases in KOOS values represent improvement. Patients reported a non-significant $(\mathrm{P}=0.25)$ improvement on the knee Pain KOOS subscale after 8 weeks of exercise ( small effect $=0.36$ mean $=4.9,95 \% \mathrm{CI}-2.7-12.5$ ) suggesting possible pain reduction of questionable clinical importance. Patients reported a significant change on the KOOS symptoms and stiffness (moderate effect (0.50), mean $=8.1,95 \%$ CI 1.5 $14.8, \mathrm{P}=0.02$ ) suggesting a possible clinically meaningful benefit. Patients reported improved Function on the KOOS subscale (large effect (0.80, Mean $=10.6$ 95\% CI -1.2 - 9.6), $\mathrm{P}=0.12$ ) however much of the perceived improvement (6.8 of 10.6 point improvement) came between the first and second baseline measurements. Nonetheless, improvement did occur from the second baseline measure to the final measure ( mean $=3.8,95 \% \mathrm{CI}=1.1-8.8 ., \mathrm{P}=0.02$ ). Improvement was reported on the function-sports and recreation KOOS subscale scores, (moderate ((0.54) effect, mean $=11.0,95 \% \mathrm{CI}=2.4-19.6 \mathrm{P}=0.036)$ however the improvement occurred between the baseline measures. These results suggest that the participants felt they could function better after having completed the initial assessment. The perceived improvements were preserved through the end of the study. Finally, participants reported non-significant changes in QOL (small effect (0.26) mean $=3.9,95 \% \mathrm{CI}=-5.5$ $-13.4, \mathrm{P}=0.61)$.

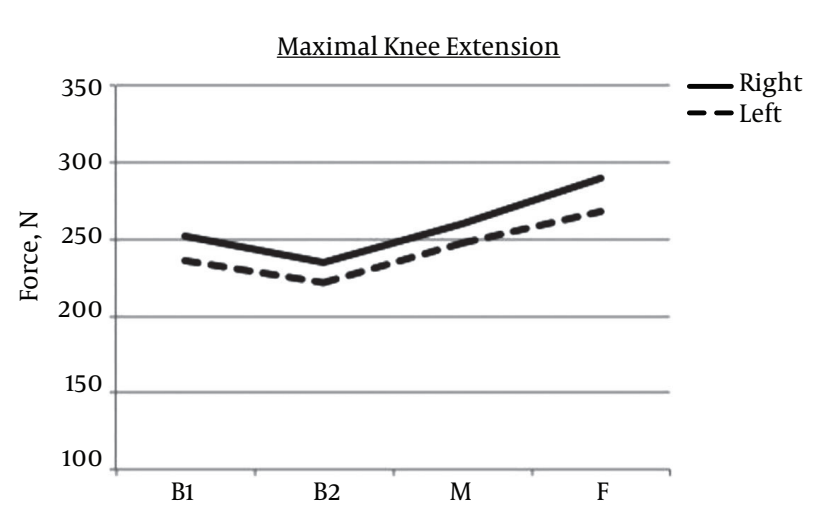

Figure 2. Maximal Knee Extension Data

\section{Discussion}

Our experience with this investigation suggests a community-based, progressive aquatic program is feasible and attractive to community dwelling adults with moderate to severe knee osteoarthritis. While three participants did not complete the program, none of the attrition was related to the exercise routine. We did not formally follow participants after the completion of the program, but several continued to exercise at the Swymfit facility on a fee basis after the study concluded.

The major findings of the study were the substantial improvements on the 2 minutes walk test and the stair climbing test. A 2 minutes walk test is commonly used as a functional measure of endurance and community function. It has been suggested that individuals need to be able to walk approximately 152 meters (500 feet) within 2 minutes for effective community ambulation (10). At baseline, $35 \%$ of our participants walked less than 152 meters. However, upon study completion, all of participants we able to walk more than 171 meters (560 feet), indicating the ability for all participants to participate in ambulation within their communities.

The 10 steps stair climb is related directly to one of the most pain provoking functional activities that those with knee OA must tolerate each day. Lower body strength and functional ability are required to ascend 10 stairs. Participants were asked to ascend the stairs as fast as possible. Participants were significantly faster after 4 and 8 weeks of 


\begin{tabular}{|c|c|c|c|c|c|c|c|c|c|c|c|c|c|c|c|c|c|}
\hline \multicolumn{18}{|c|}{ Baseline Values by Outcome Measure (Mean \pm SD) } \\
\hline \multicolumn{2}{|c|}{ 2MWT } & \multicolumn{2}{|c|}{$10 \mathrm{SSC}$} & \multicolumn{2}{|c|}{ IKESR } & \multicolumn{2}{|c|}{ IKESL } & \multicolumn{2}{|c|}{ KоOS-sS } & \multicolumn{2}{|c|}{ KOOS-P } & \multicolumn{2}{|c|}{ KOOS-FDL } & \multicolumn{2}{|c|}{ KOOS-FS } & \multicolumn{2}{|c|}{ KOOS-QOL } \\
\hline B1 & B2 & B1 & B2 & B1 & B2 & B1 & B2 & B1 & B2 & B1 & B2 & B1 & B2 & B1 & B2 & B1 & B2 \\
\hline $\begin{array}{c}158 \pm \\
23\end{array}$ & $\begin{array}{c}162 \pm \\
16\end{array}$ & $\begin{array}{l}4.98 \\
\pm 1.4\end{array}$ & $\begin{array}{c}4.67 \\
\pm 1.82\end{array}$ & $\begin{array}{c}252 \pm \\
131\end{array}$ & $\begin{array}{c}235 \pm \\
107\end{array}$ & $\begin{array}{c}236 \pm \\
107\end{array}$ & $\begin{array}{c}222 \pm \\
97\end{array}$ & $\begin{array}{c}64 \pm \\
15\end{array}$ & $\begin{array}{c}65 \pm \\
12\end{array}$ & $\begin{array}{c}62 \pm \\
16\end{array}$ & $\begin{array}{c}61 \pm \\
13\end{array}$ & $\begin{array}{c}62 \pm \\
17\end{array}$ & $\begin{array}{c}69 \pm \\
11\end{array}$ & $\begin{array}{c}22 \pm \\
19\end{array}$ & $\begin{array}{c}33 \pm \\
22\end{array}$ & $\begin{array}{c}37 \pm \\
21\end{array}$ & $\begin{array}{c}45 \pm \\
14\end{array}$ \\
\hline
\end{tabular}

Abbreviations: B1, baseline 1; B2, baseline 2; 2MWT, meters covered in 2 minutes; 10SSC, seconds to ascend 10 steps; IKES, isometric knee extension strength in Newtons; KOOS, knee injury and osteoarthrisis outcome score; SS, symptom and stiffness; P, pain; FDL, function of daily living; FS, function of sport; QOL, quality of life.

aquatic exercise. The magnitude of improvement cannot be interpreted against standard values or reported clinically important differences due to the nonstandard stair height at the Swymfit facility.

Isometric knee extension strength was obtained as a measure of quadriceps muscle strength. Quadriceps muscle strength has been linked to the ability to rise from a chair, (11), climb stairs (12) and function in the community. The force generated by the quadriceps decreased between the 1st and 2nd baseline measures. However, at midpoint measurement, participants had returned to baseline values and exceeded baseline values at the end of their exercise program. The initial decrease in strength was likely due to joint and muscle pain caused by the unaccustomed maximal effort during testing. However, the small improvements at the end of the program indicate the progressive aquatic program was building quadriceps strength. We can only speculate on the effects of a longer program, but it is known that muscle force development will continue to improve beyond 8 weeks of resistance training.

The KOOS instrument provides a means of self-report on 5 aspects of a knee osteoarthritis patient's daily experiences. Our participants reported improvement in their symptoms and stiffness, which suggests a clinically meaningful benefit. There were also improvements of daily living function, as well as sports and recreation function, indicating participants rated themselves as better able to tolerate both daily and recreational activities. As noted in the results however, the bulk of improvement in selfreported function occurred between the baseline measurements suggesting that the completion of testing altered the participants' perception of their abilities. These improvements were maintained throughout the exercise program.

Although the average decrease in reported pain was not statistically significant, less pain was reported after completion of the aquatic exercise program suggesting possible clinical benefit (13). Moreover, these data suggest that the increase in physical activity associated with the exercise program and the noted improvements in performance were not associated with an increase in knee pain.
We believe that an investigation of the effects of a longer progressive exercise program on pain is warranted.

There was not a significant improvement in selfreported QOL; however, there also was no decrease in quality of life sub-classification scores. These results suggest that a patient's OA associated symptoms and stiffness, as well as walking and climbing ability are among many factors within the multi-faceted aspects that impact selfreported QOL. We are confident that the exercise program did not diminish quality of life. The continuation of participants in an exercise program after the completion of the study suggests that our participants valued the experience and hoped to make further gains through regular, long-term participation.

This study is not without limitations. We chose not to include a control group because the study offered participants no-cost access of up to 10 weeks of supervised aquatic exercise training. Data from control groups of recent studies (14-17) involving osteoarthritis and exercise were reviewed. Improvements in function were noted in both land-based strengthening programs and aquatic-based exercise programs; however, the authors of each study discussed a lack of improvement in control groups on all outcomes measures. The improvements we observed in measures of walking distance and stair climbing are objective findings. We acknowledge the potential for bias in selfreport measures. Given that existing literature suggests that little change would be expected in a control group and that the improvements in self-report measures correspond to the functional improvements noted, we believe these data represent real change. In acknowledging the limitations of our work it is also important to note that the program was free of charge for a limited period of time. The addition of a control group would have precluded so volunteers from receiving the potential benefits of the exercise program. We choose equipoise over rigid control to maximize the opportunities for our volunteers.

Scheduling did not permit extending the duration of the study and thus we choose to allow all volunteers to begin the exercise training once they were cleared by their physician and completed baseline testing. It is unlikely that the observed improvements in 2-minute walk perfor- 
mance or stair climbing were related to placebo or the natural history of knee OA, which typically worsens over time. While self-reported improvements could occur due to a feeling of improvement or desire to report anticipated benefits, the functional improvements reported correspond to the improvements in walking and stair climbing ability. The more modest changes in pain also suggest that the self-report values reflect real functional change.

As noted, the stair height at the Swymfit Inc. facility was $14.2 \mathrm{~cm}$ rather than a standard $20.3 \mathrm{~cm}$. This difference did not affect our ability to detect improvement, as testing was consistent across all participants during all assessment sessions. The magnitude of improvement however might have been different using a standard stair height and cannot be compared to changes in stair climbing performance reported in studies where stair height was a standard $20.3 \mathrm{~cm}$ (8 inches).

In summary, an 8 - 10 week progressive aquatic exercise program for community dwelling adults with Grade II/III knee osteoarthritis is feasible and can improve community and self-reported function and reduce symptoms and stiffness, without exacerbating pain. The impact of a longer training period, particularly as related to selfreported pain, as well as longer follow up warrants further investigation.

\section{Supplementary Material}

Supplementary material(s) is available here.

\section{Acknowledgments}

We wish to thank Mr. Curtis Schultz for coordinating participant scheduling and supervision of several of the exercise sessions.

\section{Footnotes}

Authors' Contribution: Study concept and design, Craig R Denegar; acquisition of data, Dayle Maryanna Masslon, Allison Rodgers, Amelia Foster, Megan Kennedy, Aki Takahashi, Steve Victorson, Michael Joseph, Craig R Denegar; analysis and interpretation of data, Dayle Maryanna Masslon, Craig R Denegar; drafting of the manuscript, Dayle Maryanna Masslon, Craig R Denegar, Aki Takahashi, Steve Victorson; critical revision of the manuscript for important intellectual content, Dayle Maryanna Masslon, Craig R Denegar, Steve Victorson; statistical analysis, Craig R Denegar; administrative, technical, and material support, Dayle Maryanna Masslon, Craig R Denegar, Steve Victorson, Aki Takahashi, Allison Rodgers; study supervision: Craig R Denegar.
Declaration of Interest: Dr. Victorson is the owner of Swymfit Inc. but received no compensation or promotional benefit from his work in this research. No other potential conflicts of interest relevant to this article exist and the authors report no additional declaration of interest. The authors alone are responsible for the content and the writing of the paper.

\section{References}

1. Sacks JJ, Luo YH, Helmick CG. Prevalence of specific types of arthritis and other rheumatic conditions in the ambulatory health care system in the United States, 2001-2005. Arthritis Care Res (Hoboken). 2010;62(4):460-4. doi: 10.1002/acr.20041. [PubMed: 20391499].

2. Prieto-Alhambra D, Judge A, Javaid MK, Cooper C, Diez-Perez A, Arden NK. Incidence and risk factors for clinically diagnosed knee, hip and hand osteoarthritis: influences of age, gender and osteoarthritis affecting other joints. Ann Rheum Dis. 2014;73(9):1659-64. doi: 10.1136/annrheumdis-2013-203355. [PubMed: 23744977].

3. Dillon CF, Rasch EK, Gu Q, Hirsch R. Prevalence of knee osteoarthritis in the United States: arthritis data from the Third National Health and Nutrition Examination Survey 1991-94. J Rheumatol. 2006;33(11):22719. [PubMed: 17013996].

4. McAlindon TE, Bannuru RR, Sullivan MC, Arden NK, Berenbaum F, Bierma-Zeinstra SM, et al. OARSI guidelines for the nonsurgical management of knee osteoarthritis. Osteoarthritis Cartilage. 2014;22(3):363-88. doi: 10.1016/j.joca.2014.01.003. [PubMed: 24462672].

5. Fransen M, McConnell S, Harmer AR, Van der Esch M, Simic M, Bennell KL. Exercise for osteoarthritis of the knee. Cochrane Database Syst Rev. 2015;1:CD004376. doi: 10.1002/14651858.CD004376.pub3. [PubMed: 25569281].

6. Hochberg MC, Altman RD, April KT, Benkhalti M, Guyatt G, McGowan J, et al. American College of Rheumatology 2012 recommendations for the use of nonpharmacologic and pharmacologic therapies in osteoarthritis of the hand, hip, and knee. Arthritis Care Res (Hoboken) 2012;64(4):465-74. [PubMed: 22563589].

7. Bekkers JE, de Windt TS, Raijmakers NJ, Dhert WJ, Saris DB. Validation of the Knee Injury and Osteoarthritis Outcome Score (KOOS) for the treatment of focal cartilage lesions. Osteoarthritis Cartilage. 2009;17(11):1434-9. doi: 10.1016/j.joca.2009.04.019. [PubMed: 19454278].

8. Butland RJ, Pang J, Gross ER, Woodcock AA, Geddes DM. Two-, six-, and 12-minute walking tests in respiratory disease. Br Med J (Clin Res Ed). 1982;284(6329):1607-8. [PubMed: 6805625].

9. Bohannon RW, Bubela DJ, Wang YC, Magasi SR, Gershon RC. Adequacy of belt-stabilized testing of knee extension strength. J Strength Cond Res. 2011;25(7):1963-7. doi: 10.1519/JSC.ob013e3181e4f5ce. [PubMed: 21399535].

10. Bohannon RW, Bubela D, Magasi S, McCreath H, Wang YC, Reuben D, et al. Comparison of walking performance over the first 2 minutes and the full 6 minutes of the Six-Minute Walk Test. BMC Res Notes. 2014;7:269. doi: 10.1186/1756-0500-7-269. [PubMed: 24767634].

11. Hughes MA, Myers BS, Schenkman ML. The role of strength in rising from a chair in the functionally impaired elderly. $J$ Biomech. 1996;29(12):1509-13. [PubMed: 8945648].

12. Bassey EJ, Fiatarone MA, O’Neill EF, Kelly M, Evans WJ, Lipsitz LA. Leg extensor power and functional performance in very old men and women. Clin Sci (Lond). 1992;82(3):321-7. [PubMed: 1312417].

13. Batterham AM, Hopkins WG. Making meaningful inferences about magnitudes. Int J Sports Physiol Perform. 2006;1(1):50-7. [PubMed: 19114737]. 
14. Foley A, Halbert J, Hewitt T, Crotty M. Does hydrotherapy improve strength and physical function in patients with osteoarthritis-a randomised controlled trial comparing a gym based and a hydrotherapy based strengthening programme. Annals Rheum Dis. 2003;62(12):1162-7.

15. Hinman RS, Heywood SE, Day AR. Aquatic physical therapy for hip and knee osteoarthritis: results of a single-blind randomized controlled trial. Phys Ther. 2007;87(1):32-43. doi:10.2522/ptj.20060006. [PubMed:
17142642].

16. Kim IS, Chung SH, Park YJ, Kang HY. The effectiveness of an aquarobic exercise program for patients with osteoarthritis. Appl Nurs Res. 2012;25(3):181-9. doi: 10.1016/j.apnr.2010.10.001. [PubMed: 21193289].

17. Lim JY, Tchai E, Jang SN. Effectiveness of aquatic exercise for obese patients with knee osteoarthritis: a randomized controlled trial. PM R. 2010;2(8):723-31. doi: 10.1016/j.pmrj.2010.04.004. [PubMed: 20709301] quiz 793. 
Table 1. Progressive Aquatic Exercise Program

\begin{tabular}{|c|c|c|}
\hline & Warm-up Sessions (10 - 15 minutes) & Specific Strength Exercise (35 - 40 minutes) \\
\hline \multirow{6}{*}{ Week 1-Sessions 1, 2, and 3} & \multirow{6}{*}{$\begin{array}{l}\text { Learn basic movement patterns; Learn proper body } \\
\text { mechanics; *Continue Week 1; Warm up before each } \\
\text { session }\end{array}$} & Forward/backward walking -double/alternate arms \\
\hline & & Short and long strides \\
\hline & & Vertical stretch (w/long barbells) \\
\hline & & Standing squats (w/long barbells) \\
\hline & & Sitting on long barbell (leg extensions + leg curls) \\
\hline & & Vertical jogging (long barbell dragging) \\
\hline \multirow{3}{*}{ Week 2 - Session 1} & & Strength exercises from week 1 (60\% RPE) \\
\hline & & Progress from vertical jog w/ barbell dragging \\
\hline & & Barbell squats (Focus on balance and knee ROM) \\
\hline \multirow{3}{*}{ Week 2-Session 2} & & Strength exercises from week 1 (60\% RPE) \\
\hline & & Power strides (long-reaching strides) \\
\hline & & Power running ( 4 steps $50 \% \mathrm{RPE} \rightarrow 4$ steps $70 \% \mathrm{RPE}$ ), legs only \\
\hline \multirow{4}{*}{ Week 2-Session 3} & & Strength Exercises from Week 1 (60\% RPE) \\
\hline & & Sitting on barbell (without arms) \\
\hline & & Vertical jogging with barbell ( 4 Slow 50\% RPE $\rightarrow 4$ Fast 70\% RPE) \\
\hline & & Barbell squats \\
\hline \multirow{3}{*}{ Week 3 - Sessions 1, 2, and 3} & & Strength exercises from week 2 \\
\hline & & (Work to steady rate $70 \%-80 \%$ RPE) \\
\hline & & Straight leg kicks in vertical position (60\% RPE) \\
\hline \multirow{3}{*}{ Week 4 - Session 1} & & Power strides (long reaching strides) \\
\hline & & Power running ( 4 steps 50\% RPE $\rightarrow 4$ steps 70\% RPE) \\
\hline & & Prone bent knee double/alternate knee kicks (60\% RPE) \\
\hline \multirow{4}{*}{ Week 4 - Session 2} & & Easy Day \\
\hline & & Stretching under water (60\% RPE Max) \\
\hline & & Focus strictly on form and position \\
\hline & & *Use all previous exercises \\
\hline \multirow{2}{*}{ Week 4 - Session 3} & & Repeat Week 4 (Session 1 ) \\
\hline & & Prone double/alt knee kicks ( 4 Slow $50 \%$ RPE $\rightarrow 4$ Fast 70\% - 80\% RPE) \\
\hline \multirow{3}{*}{ Week 5 - Sessions 1, 2, and 3} & & Repeat Week 2 exercises \\
\hline & & Perform all exercises with small ankle cuffs \\
\hline & & *Focus on Strength \\
\hline \multirow{3}{*}{ Week 6 - Sessions 1, 2, and 3} & & Repeat Week 3 exercises \\
\hline & & Perform all exercises with small ankle cuffs \\
\hline & & *Focus on Strength \\
\hline \multirow{5}{*}{ Week 7-Session 1} & \multirow{5}{*}{ *Perform warm-up with Water-Walkers, 50\% RPE } & Remove small ankle cuffs for all exercises \\
\hline & & Vertical jogging combination \\
\hline & & High knee stride \\
\hline & & Lateral steps \\
\hline & & Combination (High knee stride + Lateral steps) \\
\hline
\end{tabular}




\begin{tabular}{|c|c|}
\hline & Power running (60\% - 70\% RPE) \\
\hline \multirow{3}{*}{ Week 7-Session 2} & Power strides (long reaching strides) \\
\hline & Power running ( 4 steps forward $\rightarrow 4$ steps sprinting in place) \\
\hline & Straight leg kicks \\
\hline \multirow{6}{*}{ Week 7-Session 3} & Use water-walkers for all exercises \\
\hline & Vertical jogging ( 4 steps 50\% RPE $\rightarrow 4$ steps 70\% RPE) \\
\hline & High knee stride \\
\hline & Lateral steps \\
\hline & Combination (High knee stride + Lateral steps) \\
\hline & Horizontal jogging ( 4 steps 50\% RPE $\rightarrow 4$ steps 70\% RPE) \\
\hline \multirow{5}{*}{ Week 8 - Session 1} & Use small ankle cuffs for all exercises (Steady state - 70\% RPE) \\
\hline & Sitting on barbell (forward/backward) \\
\hline & Vertical jogging (Barbell dragging) \\
\hline & Power strides \\
\hline & Straight leg kicks \\
\hline \multirow{4}{*}{ Week 8 - Session 2} & Remove small ankle cuffs for all exercises (Steady state - $70 \%$ RPE) \\
\hline & Seated barbell (Forward/Backward) \\
\hline & Alternate/double knee kicks \\
\hline & Barbell squats \\
\hline \multirow{5}{*}{ Week 8 - Session 3} & Use water walkers with small ankle cuffs (Steady state - 70\% RPE) \\
\hline & Vertical/Horizontal jogging \\
\hline & High knee strides \\
\hline & Lateral steps \\
\hline & Combination (high knee strides + Lateral steps) \\
\hline
\end{tabular}

